\title{
Effect of resumption of second line drugs in patients with rheumatoid arthritis that flared up after treatment discontinuation
}

\author{
Saskia ten Wolde, Jo Hermans, Ferdinand C Breedveld, Ben A C Dijkmans
}

Departments of Rheumatology $\mathrm{S}$ ten Wolde

F C Breedveld

and Medical Statistics J Hermans

University Hospital Leiden, the Netherlands

Department of Rheumatology of Free University Hospital Amsterdam, the

Netherlands

B A C Dijkmans

Correspondence to:

Dr S ten Wolde, Department of Rheumatology, University Hospital Leiden, Building 1, C4R, PO Box 9600, 2300

RC Leiden, the Netherlands.

Accepted for publication 14 January 1997

\begin{abstract}
Objective-To assess the effect of resumption of second line drugs in patients with rheumatoid arthritis (RA) that flared after treatment discontinuation.

Methods-RA patients were studied whose RA flared up after discontinuation of second line treatment while being in remission and who received a second course of the drug. Disease activity parameters were prospectively assessed at the time of treatment discontinuation, during the period when the disease flared up, and three months thereafter. Furthermore the medical charts were reviewed at 12 months after treatment resumption.
\end{abstract}

Results-There were 51 patients included in the study: 25 patients treated with antimalarial drugs, 10 with parenteral gold, four with d-penicillamine, eight with sulphasalazine, two with azathioprine, and two with methotrexate. Disease activity parameters showed significant improvement within three months of treatment resumption, but remained significantly worse when compared with that measured before treatment discontinuation. Within three months $47 \%$ of the patients fulfilled $20 \%$ response criteria. Disease activity 12 months after treatment resumption was considered to be absent in $35 \%$, mild in $43 \%$, and moderate or active in $22 \%$ of the patients. In four $(8 \%)$ patients the resumed treatment was stopped because of lack of efficacy. Side effects were recorded in four patients, which did not result in treatment discontinuation.

Conclusions-Resumption of second line drugs in RA patients whose disease flared up after discontinuation of treatment is effective and safe in most patients. Half of the patients responded within three months after resumption of the second line drug.

(Ann Rheum Dis 1997;56:235-239)

Patients with rheumatoid arthritis (RA) who favourably respond to second line antirheumatic drugs confront the physician with the dilemma whether continuation or discontinuation of the treatment should be advised. Arguments in favour of the first option came from studies that showed an increased risk of flare of the disease after discontinuation of second line drugs in RA patients with inactive disease, and a relatively low risk of side effects during long term continuous treatment. ${ }^{1-5}$ Despite the increased risk, however, many patients will not experience a flare up of the disease after treatment has finished and if they do several studies suggest a good therapeutic response to resumption of the interrupted antirheumatic therapy. $^{2-46-12}$ A recently completed placebo controlled study of discontinuation of second line drugs in RA patients who were in clinical remission gave us the opportunity to study this dilemma in more detail. ${ }^{13}$ Thirty eight per cent of the patients experienced a flare up of the disease in the first year following discontinuation, which was significantly more frequent than the $22 \%$ whose disease flared up during continuous treatment. To confirm this finding additional information on the course of the period of the flare up of the disease is needed. If patients who experience a flare up of the disease show prompt improvement after treatment resumption, discontinuation of second line treatment during remission might be regarded as an attractive alternative. Therefore, in this study, we investigated the effect of treatment resumption of second line drugs in patients whose disease flared up after the end of the treatment.

\section{Methods}

SELECTION OF PATIENTS

Patients included in the study had participated in a placebo controlled discontinuation study to compare the flare risk during continuous treatment with that after discontinuation of long term second line drug therapy during remission. ${ }^{13}$ Patients had been eligible for the randomised study if they had met the following five criteria: (a) age between 18 and 85 years, (b) RA according to the 1987 criteria, ${ }^{14}$ (c) inactive disease according to modified ARA criteria for remission, ${ }^{15}$ (d) stable disease for at least one year according to the patient's chart, (e) well tolerated treatment with one of the following second line drugs for at least the past two years: chloroquine, hydroxychloroquine, parenteral gold (aurothioglucose in oil), d-penicillamine, sulphasalazine, azathioprine or methotrexate. Exclusion criteria had been prednisone use or a previous unsuccessful attempt to stop the second line drug.

All patients who developed a flare of their disease while being in the placebo arm of the placebo controlled discontinuation study and resumed the same second line drug as before were included in the study. A flare up of the disease had been defined as the recurrence of 
synovitis such that discontinuation of the protocol was considered necessary. It was strongly advised to the treating rheumatologist to treat the patient whose disease flared up with the same second line drug as before the start of the placebo controlled study. It should be emphasised that this study is observational and not a double blind placebo controlled study.

FOLLOW UP ASSESSMENTS

At the moment of the flare up of the disease and three months thereafter the patients were assessed by one observer. The following demographic and disease characteristics had been recorded: sex, age, disease duration of RA, the presence of erosions on hand and foot radiographs and an ever positive rheumatoid factor (RF) at entry of the placebo controlled study. The following variables of disease activity were measured: (a) anamnestic pain at rest and on active movement on a four point scale $(0=$ no pain, $1=$ mild, $2=$ moderate, and $3=$ severe); (b) fatigue scored on a four point scale $(0=$ no fatigue, $1=$ mild, $3=$ moderate , and $3=$ severe); (c) duration of morning stiffness (minutes); (d) grip strength; (e) joint examination for pain using the Ritchie articular index ${ }^{16}$ and for soft tissue swelling, whereby groups of joints, for example, metacarpophalangeal or proximal joints, were considered as one joint on each side (maximum number of swollen joints was therefore 22); (f) functional ability measured by a Health Assessment Questionnaire (HAQ); (g) laboratory tests included a complete blood count, erythrocyte sedimentation rate (ESR), and serum $\mathrm{C}$ reactive protein (CRP) concentration. Furthermore at the moment of second line drug discontinuation and the moment of second line drug resumption treatment with non-steroidal anti-inflammatory drugs (NSAIDs) was recorded.

Twelve months after the development of a flare up of the disease and treatment resumption the second line drug therapy and the doctor's judgement of the whole of the disease activity (remission, mild, moderate or severe disease activity) were recorded.

During the first three months of follow up the patient was asked whether possible side effects had been noticed and safety as measured with laboratory tests were also registered. At 12 months the adverse events registered in the medical chart were recorded.

To assess the number of individual patients that improved, modified Paulus criteria ${ }^{17}$ for $20 \%$ improvement were used. A responder to treatment resumption experienced $20 \%$ or more improvement in three of the following four disease activity parameters: Ritchie articular index, number of swollen joints, ESR, and duration of morning stiffness, and at least one point improvement on the four point scale for anamnestic pain at rest, or on active movement, or both.

As the response to treatment resumption could have been influenced by the severity of a flare up of the disease the following criteria were used for a severe flare: (1) three or more swollen joints and (2) two or more of the following three criteria: (a) Ritchie articular index of more than 9; (b) duration of morning stiffness more than 45 minutes; (c) ESR of more than $28 \mathrm{~mm} / 1 \mathrm{st} \mathrm{h}$ for men and $38 \mathrm{~mm} /$ 1 st $\mathrm{h}$ for women.

STATISTICAL ANALYSIS

Changes in the disease activity parameters of patients treated with a second course of the second line drug therapy that had been stopped were analysed with a paired Student's $t$ test and Wilcoxon signed rank test for the following comparisons: (a) between the moment of flare and three months after treatment resumption, and (b) between the moment of treatment discontinuation and three months after treatment resumption.

To analyse the relation between the duration of discontinued treatment in weeks and the occurrence of a response three months after treatment resumption a Mann-Whitney $U$ test was performed.

\section{Results}

PATIENT POPULATION

A flare up of the disease had occurred in 53 RA patients while being in the placebo arm of the placebo controlled discontinuation study (fig 1). Table 1 summarises the demographic and disease characteristics of these 53 patients. At the baseline of the placebo controlled study the continued-treatment and placebo groups were well balanced with respect to demographic and disease characteristics. ${ }^{13}$ Twenty four of the 53 patients fulfilled the criteria for a severe flare. A second course of the second line drug therapy previously interrupted was prescribed in 51 patients (table 2). Two patients with a severe flare up of the disease did not resume the drugs they were treated with before the placebo controlled study; one patient improved sufficiently with NSAID therapy, and one patient preferred to start with sulphasalazine instead of parenteral gold. In $26(51 \%)$ patients the dose of the prescribed drug was higher after the flare up of the disease compared with the dose before treatment discontinuation. None of the patients was treated with prednisone. NSAIDs were newly started in 12 of 51 patients after the development of a flare. In total 37 of 51 patients were treated with NSAIDs after second line drug resumption.

EFFECT OF RESUMPTION OF SECOND LINE DRUGS Analysis of 51 patients treated with a second course of second line therapy showed that almost all disease activity parameters improved

Table 1 Characteristics of 53 patients with $R A$ whose disease flared after discontinuation of second line treatment while being in remission

\begin{tabular}{ll}
\hline Characteristic & \\
\hline Age (years) & \\
Female (\%) & $59.9(11.4)$ \\
Duration of RA (years) & 60 \\
ELISA IgM-RF positive (\%) & $10.6(9)$ \\
Erosive changes on $x$ ray (\%) & 77 \\
Weeks between entry in the placebo controlled & 75 \\
$\quad$ study and flare & 27 (3 to 52) \\
Number of patients with severe flare & 24 \\
\hline
\end{tabular}

Data shown as mean $(\mathrm{SD})^{\star}$ and median (range) $\dagger$. 
Table 2 Type and dose of treatments of 51 patients with $R A$ who resumed second line drug treatment after discontinuation because of remission

\begin{tabular}{lll}
\hline Second line drug & Number of patients & Median dose of resumed treatment (range) \\
\hline Chloroquine & 10 & $700(400$ to 1400$) \mathrm{mg} / \mathrm{week}$ \\
Hydroxy-chloroquine & 15 & $2800(1400$ to 4200$) \mathrm{mg} / \mathrm{week}$ \\
Parenteral gold & 10 & $1300(130$ to 2600$) \mathrm{mg} / \mathrm{year}$ \\
d-Penicillamine & 4 & $250(250$ to 600$) \mathrm{mg} / \mathrm{day}$ \\
Sulphasalazine & 8 & $2000(1500$ to 2000$) \mathrm{mg} / \mathrm{day}$ \\
Azathioprine & 2 & $125(100$ to 150$) \mathrm{mg} / \mathrm{day}$ \\
Methotrexate & 2 & $7.5(7.5$ to 7.5$) \mathrm{mg} / \mathrm{week}$ \\
\hline
\end{tabular}

significantly within three months (table 3 ). Comparison of the disease activity parameters at the start of the placebo controlled study, ${ }^{13}$ when the patients fulfilled modified ARA criteria for remission, ${ }^{15}$ with those three months after treatment resumption showed that at that time the patients still showed significantly higher levels of disease activity than at the moment treatment had been stopped (table 3).

According to the $20 \%$ response criteria 24 $(47 \%)$ patients could be designated as responders within three months after treatment resumption. Separate analysis of these 24 responders showed that within three months after treatment resumption $50 \%$ of these patients achieved the same level of disease activity parameters as before treatment discontinuation concerning duration of morning stiffness, Ritchie articular score, and number of swollen joints. Only $25 \%$ of the responders had achieved the same level of ESR again.

There was no correlation between severity of the flare and the occurrence of a response: 10 $(45 \%)$ of 22 patients with a severe flare up of the disease and $14(48 \%)$ of 29 patients with a mild flare experienced a response. There was also no relation between the duration of treatment discontinuation and the occurrence of a response three months after treatment resumption. Furthermore there was no difference in the response rate between patients who newly started NSAID therapy together with the resumption of the second line drug and the patients without change in NSAID therapy; six (50\%) of 12 patients who

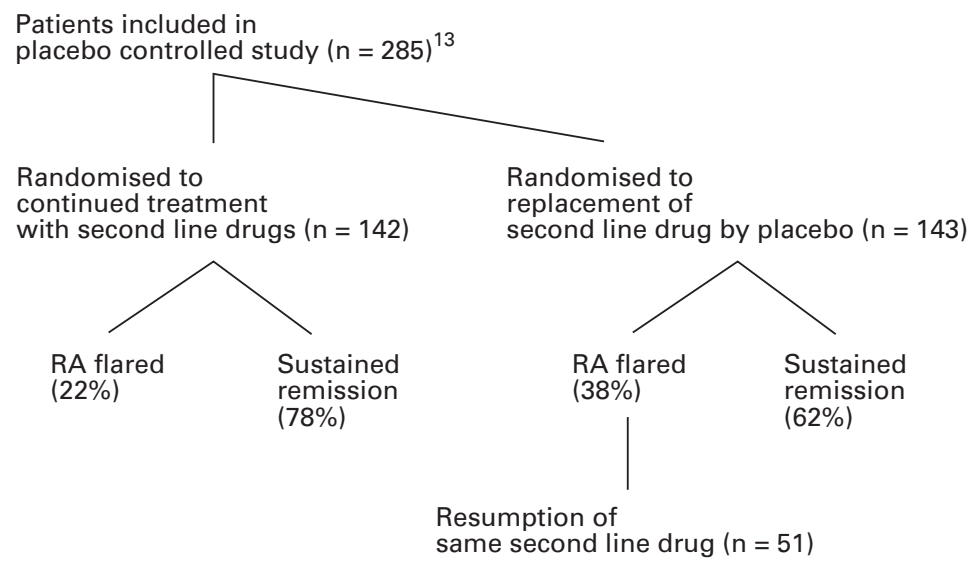

Figure 1 Flow diagram illustrating patient numbers and percentages at all stages of the placebo controlled treatment discontinuation study $y^{13}$ until the inclusion of 51 patients whose disease flared into the present second line treatment resumption study. started NSAIDs and $18(46 \%)$ of 39 patients in whom NSAIDs were either not started or continued.

To assess possible differences in effect of treatment resumption between the different second line drugs the percentages of patients fulfilling $20 \%$ response criteria three months after treatment resumption were compared for each drug: (a) antimalarials: $60 \%$ (15 of 25), (b) sulphasalazine: $63 \%$ (5 of 8 ), (c) methotrexate: $100 \%$ (2 of 2), (d) azathioprine: $50 \%$ (1 of 2 ), (e) parenteral gold: $10 \%$ (1 of 10), (f) d-penicillamine: $0 \%$ (0 of 4$)$.

OUTCOME 12 MONTHS AFTER TREATMENT RESUMPTION

Twelve months after treatment resumption 46 of 51 patients treated with a second course of second line therapy were still treated with the same drug. In four patients (two taking parenteral gold and two sulphasalazine) the second course was stopped because of lack of efficacy and patients were treated with another kind of second line therapy. One patient with an initial good response to a second course with hydroxychloroquine stopped this drug for unrecorded reasons.

Disease activity 12 months after treatment resumption was judged to be absent in 18 patients $(35 \%)$, mild in 22 patients $(43 \%)$, and moderate or severe in 11 patients $(22 \%)$.

Separate analysis for each kind of second line drug showed that the following percentages of patients regained mild or no disease activity 12 months after treatment resumption: with antimalarials $80 \%$, with parenteral gold $70 \%$, with sulphasalazine $63 \%$, with d-penicillamine $100 \%$, with azathioprine $100 \%$, and with methotrexate $100 \%$ of patients.

\section{ADVERSE EVENTS}

Side effects were registered in four patients after treatment resumption; dizziness after resumption of $d$-penicillamine $(n=1)$ and chloroquine $(n=1)$, dermatitis after resumption of parenteral gold $(n=1)$, and transient thrombocytopenia and anaemia after resumption of d-penicillamine $(n=1)$. None of these side effects has led to treatment discontinuation.

\section{Discussion}

The main conclusion of this study is that half of the RA patients whose disease flared up after discontinuation of second line treatment responded within three months after resumption of the second line drug. Half of these responders at three months were back at the disease activity level as before treatment discontinuation. Disease activity one year after treatment resumption was judged to be absent or mild in most of the patients. One year after treatment resumption $90 \%$ of the patients persistently used the resumed second line drug. In none of the patients was treatment stopped because of side effects.

Our results are in line with those of a limited number of previous studies, which suggested that a good therapeutical response to a first treatment course predicts a favourable 
Table 3 Disease activity parameters of 51 patients with $R A$ before discontinuation of second line drug treatment, at the moment of a recurrence of $R A$ immediately before treatment resumption, and three months after resumption of the same second line drug therapy

\begin{tabular}{|c|c|c|c|c|c|}
\hline Parameter & $\begin{array}{l}\text { Before treatment } \\
\text { discontinuation }\end{array}$ & $\begin{array}{l}\text { During flare, before } \\
\text { treatment resumption }\end{array}$ & $\begin{array}{l}\text { Three months after } \\
\text { treatment resumption }\end{array}$ & $p$ Value $^{\star}$ & $p$ Value ${ }^{\star *}$ \\
\hline Pain at rest & $0.2(0.5)$ & $1.4(1.0)$ & $0.6(0.8)$ & 0.02 & 0.00 \\
\hline Pain on active movement & $0.7(0.9)$ & $2.0(0.9)$ & $1.3(1.0)$ & 0.00 & 0.00 \\
\hline Fatigue score & $0.1(0.5)$ & $1.5(1.1)$ & $1.0(1.1)$ & 0.00 & 0.01 \\
\hline Morning stiffness (min) & $9(18)$ & $113(133)$ & $51(97)$ & 0.00 & 0.00 \\
\hline \multicolumn{6}{|l|}{ Grip strength $(\mathrm{kPa})$} \\
\hline Right hand & $58(20)$ & $40(20)$ & $47(23)$ & 0.00 & 0.00 \\
\hline Left hand & $57(20)$ & $40(19)$ & $43(22)$ & 0.00 & 0.14 \\
\hline HAQ score & $0.5(0.6)$ & $1.0(0.8)$ & $0.7(0.8)$ & 0.00 & 0.00 \\
\hline Ritchie articular index & $2.7(2.8)$ & $10.5(5.8)$ & $7.1(6.2)$ & 0.00 & 0.00 \\
\hline Number of swollen joints & $0.8(1.0)$ & $3.8(2.3)$ & $2.9(2.2)$ & 0.00 & 0.01 \\
\hline $\operatorname{ESR}(\mathrm{mm} / 1 \mathrm{st} \mathrm{h})$ & $15(11)$ & $28(18)$ & $24(19)$ & 0.00 & 0.01 \\
\hline CRP (mg/l) & $7(7)$ & 15 (13) & $13(14)$ & 0.00 & 0.07 \\
\hline
\end{tabular}

Data shown as mean (SD). ${ }^{\star}$ p Value of Wilcoxon signed rank test for comparison between parameters measured before treatment discontinuation and three months after treatment resumption. $\nmid \mathrm{p}$ Value of Wilcoxon signed rank test for comparison between parameters measured at the time of a flare and three months after treatment resumption.

response to retreatment. ${ }^{2-47812}$ Three of these studies are retrospective studies of a second course of parenteral gold. There is one more retrospective study in which only $36 \%$ of the patients who initially were in remission during gold treatment reached this therapeutic goal again with a second course of gold. ${ }^{11}$ In the present study the response rates three months after resumption of parenteral gold are also low. However, one year after treatment resumption seven of 10 patients were considered to have mild or no disease activity. These results suggest that the response to a second course with gold is slow but effective. The response to antimalarials, sulphasalazine, azathioprine, and methotrexate seemed to start within three months after treatment resumption. The small numbers of patients in each treatment group, however, made comparisons between subgroups difficult.

The dose of the resumed second line drugs was generally lower than that recommended for patients with active disease. As this is an observational study such action most closely reflects what would happen in daily practice. Possibly the response rate would have been higher when patients were treated with the recommended doses for active disease.

The dilemma as to whether continuation or discontinuation of second line treatment during inactive disease can be advised should be considered with the following points in mind. The risk of side effects is low both for the patients treated with a second course of second line drugs and for the patients receiving long term continued second line therapy. ${ }^{13}$ Therefore the issue of side effects seems to play no major part in the decision whether or not to continue a well tolerated drug in a patient with inactive RA. Discontinuation of long term second line therapy in patients with inactive disease is associated with a doubling of the flare rate as compared with continued treatment. ${ }^{13}$ The present study shows that most patients whose disease flared up after treatment discontinuation responded to treatment resumption of the second line drug. In addition it should be realised that in the same time period about $20 \%$ of patients who continue treatment will also experience a flare. Therefore it may be concluded that the major difference between the two treatment policies is the fact that a higher percentage of patients who discontinue treatment will experience a period with increased disease activity compared with patients who continue treatment. The significance of such a flare for the long term disease outcome remains uncertain. This disadvantage of drug discontinuation has to be weighed against the positive aspects many patients experience when drug intake as well as the monitoring of side effects can be stopped and that these patients are no longer at risk of the rare serious side effects of second line drugs.

In conclusion, the results of this study show that second line treatment resumption is effective and safe in most RA patients whose disease flared up after treatment discontinuation. Half of the patients fulfilled $20 \%$ response criteria within three months after treatment resumption. These findings are helpful in designing a treatment strategy for individual patients with RA who experience a long period with inactive disease during treatment with second line drugs.

Supported by a grant from 'Het Nationaal Reumafonds' of the Netherlands (STAR 91/CR/347)

1 de Silva M, Hazleman BL. ong-term azathioprine in rheumatoid arthritis: a double-blind study. Ann Rheum Dis 1981;40:560-3.

2 Cade R, Stein G, Pickering M, Schlein E, Spooner G. Low dose, long-term treatment of rheumatoid arthritis with azathioprine. South Med J 1976;69:388-92.

3 Ahern MJ, Hall ND, Case K, Maddison PJ. D-penicillamine withdrawal in rheumatoid arthritis. Ann Rheum Dis 1984; 43:213-7.

4 Kremer JM, Rynes RI, Bartholomew LE. Severe flare of rheumatoid arthritis after discontinuation of long-term rheumatoid arthritis after discontinuation of long-term
methotrexate therapy. Am J Med 1987;82:781-6.

methotrexate therapy. Am J Med 1987;82:781-6.
5 Szanto E. Low-dose methotrexate in rheumatoid arthritis: effect and tolerance. Scand J Rheumatol 1986;15:97-102. 6 Kean WF, Anastassiades TP. Long term chrysotherapy: incidence of toxicity and efficacy during sequential time periods. Arthritis Rheum 1979;22:495-501

7 Sagransky DM, Greenwald RA. Efficacy and toxicity of retreatment with gold salts: a retrospective review of 25 cases. J Rheumatol 1980;7:474-8.

8 Bagnall AW. The value of chloroquine in rheumatoid disease: a four-year study of continuous therapy. Can Med Assoc J 1957;77:182-94.

9 Jones E, Jones JV, Woodbury JF. Response to sulfasalazine in rheumatoid arthritis: life table analysis of a 5-year followup. J Rheumatol 1991;18:195-8.

10 Hoh KF, Dwosh IL, Ford PM, Anastassiades TP, Kelly HG. Drug-related disease remissions in rheumatoid arthritis. Arthritis Rheum 1981;24(suppl 72):86.

11 Evers AE, Sundstrom WR. Second course gold therapy in the treatment of rheumatoid arthritis. Arthritis Rheum 1983;26:1071-5

12 Klinkhoff AV, Teufel A. The second course of gold. J Rheumatol 1995;22:1655-66. 
13 ten Wolde S, Breedveld FC, Hermans J, Vandenbroucke JP, van de Laar MAFJ, Markusse HM, et al. Randomised placebo-controlled study of stopping second-line drugs in rheumatoid arthritis. Lancet 1996;347:347-52

14 Arnett FC, Edworthy SM, Block DA. The American Rheumatism Association 1987 revised criteria for the classification of rheumatoid arthritis. Arthritis Rheum 1988; 31:315-24

15 Pinals RS, Masi AT, Larsen RA. Preliminary criteria for clinical remission in rheumatoid arthritis. Arthritis Rheum 1981;24:1308-15.
16 Ritchie DM, Boyle JA, McInnes JM, Jasani MK, Dalakos TG, Grieveson P, et al. Clinical studies with an articular index for the assessment of joint tenderness in patients with rheumatoid arthritis. Q J Med 1968;37:393-406.

17 Paulus HE, Egger MJ, Ward JR, Williams HJ. Analysis of improvement in individual rheumatoid arthritis patients treated with disease-modifying antirheumatic drugs, based on the findings in patients treated with placebo. The Cooperative Systematic Studies of Rheumatic Diseases Group. Arthritis Rheum 1990;33:477-84. 